\title{
A Judicialização de leitos de Unidade de Terapia Intensiva: Uma visão sobre as notas técnicas do sistema NATJUS
}

\author{
The Judicialization of Intensive Care Unit Beds: An Overview of NATJUS Notes \\ Techniques
}
La judicialización de las camas de la Unidad de Cuidados Intensivos: una visión sobre las notas técnicas de NATJUS

Juliana Teixeira Gomes ${ }^{1}$, Daniel Ghiraldelli², Luis Eduardo Miani Gomes²*.

\begin{abstract}
RESUMO
Objetivo: Identificar a atuação do Núcleo de Apoio Técnico ao Judiciário (NATJUS) nos requerimentos de pareceres referente à solicitação de internação em Unidade de Terapia Intensiva. Métodos: Trata-se de um estudo quantitativo que utilizou dados secundários sobre o requerimento de parecer no sistema do Núcleo de Apoio Técnico do Poder Judiciário, referentes às solicitações de internação em unidade de terapia intensiva e cuja data de criação do parecer era referente ao ano de 2020. Resultados: Foram encontrados 104 pareceres com o descritor "unidade de terapia intensiva" no ano de 2020. Observou-se que as maiorias dos pacientes eram do sexo masculino, e a média de idade era de 56 anos. Além disso, a maioria dos pareceres (76,92\%) foi favorável às solicitações de internações, e o Estado de Tocantins foi o que mais solicitou pareceres ao núcleo de apoio técnico. Conclusão: Embora a judicialização se mostre crescente, observou-se baixo número de consultas dos Estados brasileiros diante das necessidades de internação em Unidade de Terapia Intensiva, e cujo parecer pode ofertar elementos técnicos e literários.
\end{abstract}

Palavras-chave: Judicialização em saúde, Unidade de terapia intensiva, Parecer de especialista.

\begin{abstract}
Objective: To identify the role of the Center for Technical Support to the Judiciary (NATJUS) in the requirements for opinions regarding the request for admission to the Intensive Care Unit. Methods: This is a quantitative study that used secondary data on the request for an opinion in the system of the Technical Support Nucleus of the Judiciary, referring to requests for hospitalization in an intensive care unit and whose date of creation of the opinion was related to the year 2020. Results: 104 opinions were found with the descriptor "intensive care unit" in 2020. It was observed that the majority of patients were male, and the average age was 56 years. In addition, most of the opinions (76.92\%) were favorable to requests for hospitalizations, and the State of Tocantins was the one that most requested opinions from the technical support center. Conclusion: Although judicialization is proving to be increasing, there were a low number of consultations by Brazilian states regarding the needs of hospitalization in the Intensive Care Unit, whose opinion may offer technical and literary elements.
\end{abstract}

Keywords: Judicialization in health, Intensive care unit, Expert opinion.

\section{RESUMEN}

Objetivo: Identificar el rol del Centro de Soporte Técnico al Poder Judicial (NATJUS) en los requerimientos de dictamen sobre la solicitud de ingreso a la Unidad de Cuidados Intensivos. Métodos: Se trata de un estudio cuantitativo que utilizó datos secundarios sobre la solicitud de opinión en el sistema del Núcleo de Soporte Técnico del Poder Judicial, referidos a solicitudes de hospitalización en una unidad de cuidados

1 Centro Universitário Salesiano de São Paulo (UNISAL), Americana - SP.

${ }^{2}$ Faculdade de Americana, Americana - SP. *E-mail: leduardo.adv@gmail.com

SUBMETIDO EM: 2/2021

ACEITO EM: 3/2021

PUBLICADO EM: 3/2021 
intensivos y cuya fecha de creación de la opinión estaba relacionada con año 2020. Resultados: se encontraron 104 opiniones con el descriptor "unidad de cuidados intensivos" en 2020. Se observó que la mayoría de los pacientes eran del sexo masculino y la edad promedio fue de 56 años. Además, la mayoría de las opiniones $(76,92 \%)$ fueron favorables a las solicitudes de hospitalización, y el estado de Tocantins fue el que más solicitó opiniones al centro de soporte técnico. Conclusión: Si bien la judicialización está aumentando, hubo un bajo número de consultas por parte de los estados brasileños sobre las necesidades de hospitalización en la Unidad de Cuidados Intensivos, cuya opinión puede ofrecer elementos técnicos y literarios.

Palabras clave: Judicialización en salud, Unidad de cuidados intensivos, Opinión de expertos.

\section{INTRODUÇÃO}

A saúde prevista na Constituição Federal de 1988 (C.F/1988), em especial sua descrição no artigo 196, é um direito de todos e dever do Estado, com redução do risco de doença e de outros agravos, com acesso universal e de forma igualitária a todos os cidadãos (BRASIL, 1998). Embora o direito a saúde esteja consagrado entre um dos deveres do Estado, observa-se que não vem sendo desempenhado em sua integralidade. Desse modo, o acesso à justiça como a fim de reivindicar por acessos a determinados medicamentos, insumos e atendimentos médicos hospitalares vêm sendo crescente (MADURO LCS e PEREIRA LRL, 2020).

De outro modo, observa-se que o Estado brasileiro não tem garantido as condições objetivas para a expansão econômica, por meio de políticas e ações públicas que permitam o acesso de todos ao sistema de saúde, assim como o progresso tecnológico e científico do Sistema Único de Saúde (SUS) se tornando uma caracterização amplamente conectada ao período, ao lugar e infelizmente a nível monetário que se atribui (VENTURA M, et al., 2010). Mostra-se visível a problematização que vem enfrentando Estados e Municípios (PAIM JS, 2018).

A interface entre a judicialização e as questões que envolvem a saúde vem aumentando a cada dia. Essa interface vem-se expandindo na busca de ferramentas que possam embasar as constantes discussões (LAFFIN NHF e BONACIM CAG, 2017). Com o intuito de auxiliar a prolação de decisões sob fundamento técnico científico o Conselho Nacional de Justiça (CNJ) desenvolveu uma ferramenta denominada Núcleo de Apoio Técnico ao Judiciário (NATJUS) e subsequente o banco nacional de pareceres - Sistema e-NATJUS. Assim, deixa-se de lado uma visão puramente jurídica sobre o tema (FORSTER JP, et al., 2020).

A implantação do Núcleo de Apoio Técnico ao Judiciário NATJUS foi apoiada na Resolução do Conselho Nacional de Justiça (CNJ) № 238, de 06/09/2016, ante da necessidade dos profissionais jurídicos buscarem apoio aos profissionais da área da saúde no auxílio nas soluções para as questões levadas ao Poder Judiciário pelos Tribunais de Justiça (TJs) e Tribunais Regionais Federais (TRFs) nas causas relacionadas à saúde (MARIANO CM, et al., 2018).

O NATJUS é um instrumento que está acrescentado ao sistema e-NATJUS, que igualmente fica instalado no sítio eletrônico do Conselho Nacional de Justiça. A regulamentação se deu por meio do Provimento n. 84/2019, expedido pela Corregedoria Nacional de Justiça, que dispõe sobre 0 uso e funcionamento do sistema e-NATJUS, em especial a ferramenta NATJUS. As demandas são avaliadas através de fundamentos e protocolos médicos, com base nas evidencias científicas, que fornecerão respaldo técnico para fundamentar os pareceres (CONSELHO NACIONAL DE JUSTIÇA, 2019).

Dentre as atribuições do NATJUS estão à elaboração de pareceres por profissionais de diversas Instituições de saúde. A medicina baseada em evidências é um dos pilares destes profissionais, que elaborarão pareceres que servirão de prova pericial das demandas (MARIANO CM, et al., 2018).

Os pareceres que estão sendo elaborados pelo NATJUS são provenientes de instituições de saúde e em diversas Universidades do Brasil. Além disso, os pareceres são fundamentados em protocolos ou em medicina baseada em evidencias. Importante destacar que a criação deste sistema não é para a realização 
de perícia judicial, mas, pela emissão de parecer técnico, diante de caso concreto, com emprego de protocolos e as melhores recomendações literárias (RIBEIRO IS e ANDRADE AC, 2018). Não é por demais comentar que embora o avanço das decisões judiciais, nem todas as demandas podem ser atendidos por esse sistema, tendo em vista as características individuais de cada demanda.

Algumas demandas ainda necessitam de imediata manifestação por parte do magistrado, não havendo tempo hábil para consulta e aguardar parecer técnico, embora se tenha mostrado ágil na emissão dos pareceres (FORSTER JP, et al., 2020). Conquanto todas as ações sejam no sentido de corroborar e viabilizar as decisões judiciais para a emissão de decisões de maior severidade técnica e precisão de justeza. (FORSTER JP, et al., 2020).

Outrossim, tendo em vista o gradativo de ações judiciais que envolvem pedidos de medicamentos e insumos, e ainda o acesso à internação em Unidade de Terapia Intensiva, a fundamentação e a apreciação em alguns casos é por elementos trazidos pelos autores das demandas (SANTOS FRQ, et al., 2020).

As Unidades de Terapia Intensiva - denominadas unidades especiais e específicas apresentam todo um aparato tecnológico de última geração, que necessitam de cuidados contínuos que, juntamente com a equipe multiprofissional qualificado quanto ao conhecimento técnico-científico, tem como o objetivo dentro desta unidade restabelecer parâmetros alterados aos pacientes em estado grave. Os pacientes críticos necessitam de rapidez em seu atendimento, necessitando de rigorosa atenção e cuidados mais complexos, com intuito de proporcionar melhorias e resultado positivo em seu quadro clínico (MELO EM, et al., 2014).

Observa-se ainda que em sendo a Unidade de Terapia Intensiva um setor onde a complexidade de equipamentos e dispositivos disponíveis podem salvar vidas, o seu retardamento pode trazer resultados negativos em consequência da temporização na monitorização e na admissão do tratamento mais adequado (SANTOS FRQ, et al., 2020).

Assim, o objetivo deste estudo é identificar a atuação do Núcleo de Apoio Técnico ao Judiciário (NATJUS) nos requerimentos de pareceres referente à solicitação de internação em Unidade de Terapia Intensiva.

\section{MÉTODOS}

Trata-se de um estudo quantitativo que utilizou dados secundários sobre o requerimento de parecer no sistema do Núcleo de Apoio Técnico do Poder Judiciário (NATJUS) de acesso público, para informações sobre internação em Unidade de Terapia Intensiva (UTI). Através do sistema de busca do NATJUS foram identificados os pareceres técnicos ou nota técnica cuja referência da pesquisa era o descritor "unidade de terapia intensiva".

Foram incluídos no estudo todos os pareceres que continham em seus descritores as notas referentes solicitação de internação em unidade de terapia intensiva e cuja data de criação do parecer era referente ao ano de 2020. Foram excluídos aqueles que não haviam referência aos pedidos de internação em unidade de terapia intensiva, e ano de elaboração diverso de 2020.

Foram incluídos ainda os pareceres que apresentavam em sua descrição da tecnologia ou dentro das descrições à solicitação de leito de unidade de terapia intensiva. Além disso, incluíram-se os pareceres referentes à Unidade de Terapia Intensiva Adulto, Pediátrico ou Neonatal. Os dados foram coletados do sistema de acesso público do NATJUS, dispensando aprovação do Comitê de Ética em Pesquisa conforme Resolução 510/2016 do Conselho Nacional de Saúde, por utilizar informações de banco de dados, com informações agregadas, sem possibilidade de identificação individual.

\section{RESULTADOS}

Através do sistema de busca do NATJUS foram encontrados 274 resultados com o descritor "unidade de terapia intensiva". Entretanto ao contrapor os critérios de inclusão e exclusão foram selecionados 104 pareceres. Durante o ano de 2020 foram solicitados, portanto 104 pareceres sobre a necessidade e premência de internação em UTI. A maior prevalência de solicitação foi referente ao sexo masculino (53 pareceres), e com uma média de idade de 56 anos de idade (0 - 95) (Gráfico 1). 
Gráfico 1 - Dados referentes às solicitações (sexo e faixa etária).

\begin{tabular}{rcccc} 
& Homens & Faixa & Mulheres & \\
\cline { 2 - 4 } $\mathbf{1 1 , 3 2 \%}$ & 6 & $\mathbf{0}$ a $\mathbf{1 4}$ anos & 7 & $13,73 \%$ \\
$\mathbf{5 , 6 6 \%}$ & 3 & $\mathbf{1 5}$ a $\mathbf{3 0}$ anos & 2 & $3,92 \%$ \\
$\mathbf{9 , 4 3 \%}$ & 5 & $\mathbf{3 1}$ a $\mathbf{4 4}$ anos & 10 & $19,61 \%$ \\
$\mathbf{3 0 , 1 9 \%}$ & 16 & $\mathbf{4 5}$ a $\mathbf{6 0}$ anos & 16 & $31,37 \%$ \\
$\mathbf{2 4 , 5 3 \%}$ & 13 & $\mathbf{6 1}$ a $\mathbf{7 4}$ anos & 5 & $\mathbf{9 , 8 0 \%}$ \\
$\mathbf{1 8 , 8 7 \%}$ & 10 & $\mathbf{7 5}$ a $\mathbf{9 5}$ anos & 11 & $\mathbf{2 1 , 5 7 \%}$
\end{tabular}

Fonte: Gomes JT, et al., 2021; dados extraídos do Núcleo de Apoio Técnico ao Judiciário.

Dos 104 pareceres solicitados, 80 (76,92\%) deles foram favoráveis à solicitação de internação em UTI. A justificativa de alegação de urgência, conforme definição de Urgência e Emergência do Conselho Federal de Medicina estava presente em $49(47,12 \%)$ dos pareceres. Em relação aos Estados de origem para a solicitação dos pareceres, o destaque foi para Tocantins e Bahia (67 e 26 respectivamente) (Tabela 1)

Tabela 1 - Dados referentes ao parecer favorável e Estados de origem do parecer.

\begin{tabular}{cc}
\hline Estados - origem do parecer & Parecer (quantidade) \\
\hline Bahia (BA) & 26 \\
Maranhão (MA) & 1 \\
Mato Grosso do Sul (MS) & 1 \\
Mato Grosso (MT) & 5 \\
Roraima (RR) & 1 \\
Rio Grande do Sul (RS) & 3 \\
Tocantins (TO) & 67 \\
\hline
\end{tabular}

Fonte: Gomes JT, et al., 2021; dados extraídos do Núcleo de Apoio Técnico ao Judiciário.

Ao que se refere à data da criação do parecer, observou-se uma grande incidência no mês de agosto $(41,34 \%)$ e no mês de dezembro de 2020 (25\%). As patologias descritas na emissão do parecer, e que sustentavam relação direta com as solicitações de internação na unidade de terapia intensiva foram divididas e apresentadas por sistemas. As neoplasias foram agrupadas independentes do sistema acometido (Gráfico 2).

Gráfico 2 - Dados referentes às patologias relacionadas aos pareceres.

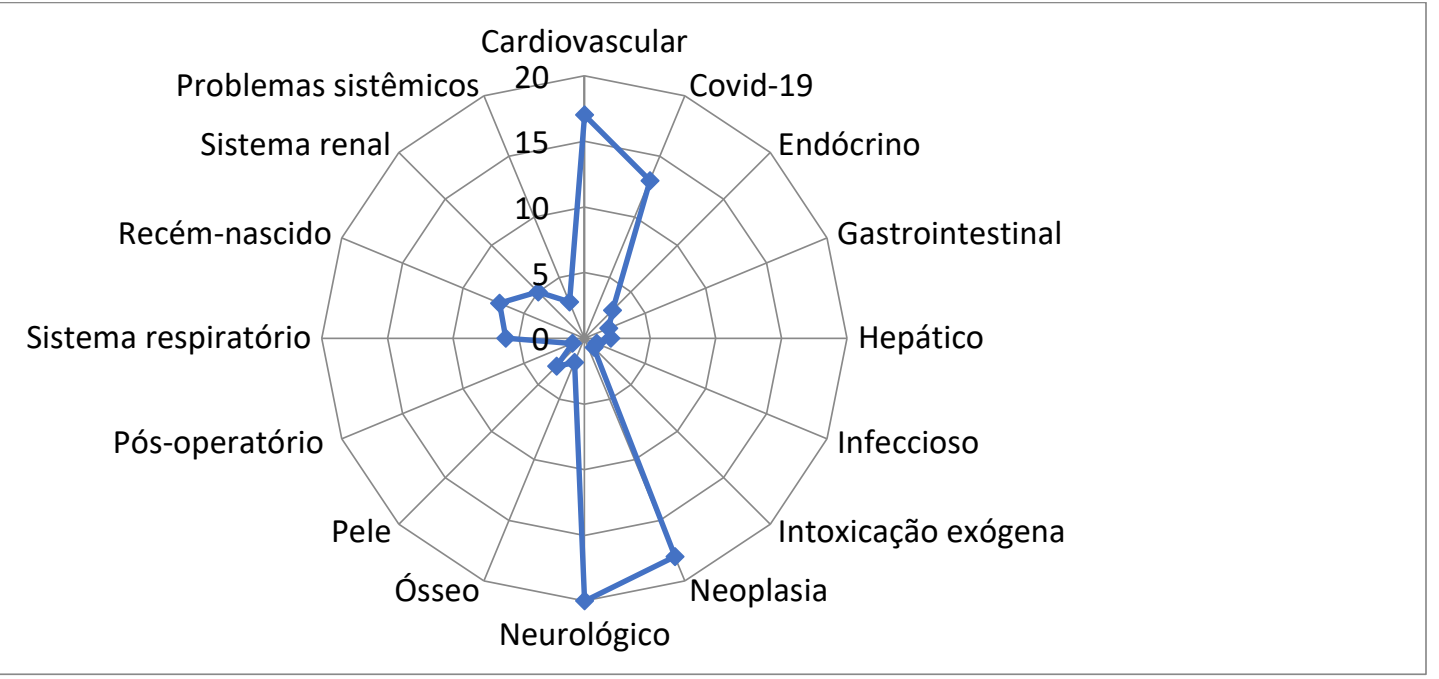

Fonte: Gomes JT, et al., 2021; dados extraídos do Núcleo de Apoio Técnico ao Judiciário. 
Em que pese às diversas patologias identificadas, observou-se que $13(12,5 \%)$ dos pedidos de internação em terapia intensiva tinha relação com a Doença da Corona Vírus (Covid-19). Entretanto, a grande maioria estava relacionada a problemas neurológicos $(19,23 \%)$ seguido de doenças cardiovasculares (16,35\%). Num perfil individualizado, em relação à Covid-19, observamos um grande número de casos acometendo o sexo masculino e no Estado do Tocantins $(84,62 \%)$ com uma média de idade de 64,1 anos. Nas doenças neurológicas nota-se a presença de trauma cranioencefálico e acidente vascular encefálico como os principais eventos.

Nas doenças cardiovasculares nota-se a presença de aneurisma e infarto agudo do miocárdio. Outrossim, com relação à conclusão do parecer sobre a solicitação da UTI observou-se que 80 (76,92\%) dos pacientes obtiveram resultados favoráveis ao pedido do parecer. Não obstante a maioria dos pareceres apresentarem favoráveis as solicitações de UTI apenas 49 (47,12\%) apresentava justificativa de urgência conforme definição de urgência e emergência do Conselho Federal de Medicina.

\section{DISCUSSÃO}

A judicialização no Brasil vem se apresentando de forma crescente. Nota-se que o acesso à justiça é um meio encontrado para se conseguir o direito a tratamento médico e/ou um acesso aos tratamentos necessários. A UTI, conforme o Ministério da Saúde é uma divisão hospitalar proposta ao atendimento de pacientes graves ou de risco, que oferece assistência médica e de enfermagem continuadas, com aparelhamentos específicos próprios e recursos humanos individualizados e particularizados, com ingresso a outras ciências dedicadas ao diagnóstico e à terapêutica necessária ao paciente (BRASIL, 1998). A ocorrência da judicialização vem se transformando em um grande problema para os cofres públicos, em especial ao cálculo governamental.

O gasto do Ministério da Saúde com medicamentos cuja provisão foi determinada por ordem judicial passou de $\mathrm{R} \$ 2,5$ milhões em 2005 para aproximadamente $\mathrm{R} \$ 266$ milhões em 2011 (ADVOCACIA GERAL DA UNIÃO, 2012). Embora em nosso estudo foram solicitados apenas 104 pareceres sobre a necessidade de internação de UTI, deste total apenas 80 deles foram favoráveis a internação. Notou-se ainda que maior incidência dos pareceres foram nos meses de agosto (41,34\%) e dezembro de 2020 (25\%). Já na década de 90 , foram encontradas ações judiciais que buscavam cirurgias, medicamentos e insumos.

Essa crescente foi anunciada pelo Ministério da Saúde, através de sua consultoria jurídica, evidenciando assim um avanço considerável de gastos públicos em relação à aquisição de medicamentos e demais insumos concedidos nas decisões (LAFFIN NHF e BONACIM CAG, 2017). A densidade média de leitos UTI no SUS e nos planos privados de assistência à saúde por 100 mil habitantes está em 21,0. Em relação aos leitos disponíveis aos habitantes dependentes do SUS apresenta-se em 13,6 e na saúde suplementar é de 62,6 (COSTA NR, 2020).

Nesse mesmo sentido, podemos destacar a disponibilidade de leitos de UTI (somados os leitos SUS e não SUS) nos cenários de taxa de ocupação de 50\%, por 100 mil habitantes em dezembro de 2019, com uma condição de disponibilidade crítica para os Estado da Bahia e Tocantins (COSTA NR, 2020). Nota-se que os Estados (Bahia e Tocantins) com maior número de solicitações de pareceres encontram-se com condição de disponibilidade crítica para leitos de UTI. Outrossim, ainda que o requerimento de pareceres ao sistema NATJUS não vincule a decisão do juiz em sua decisão, observamos que esta manifestação traz uma fundamentação mais objetiva, determinando critérios de internação em UTI de forma mais objetiva.

Ainda que positivado no ordenamento jurídico brasileiro a universidade dos serviços de saúde, aqueles realizados pelo SUS, ainda está distante e apartado daquilo que idealizado. Nesse sentido, o processo de judicialização inicia-se, a fim de buscar seu direito a saúde, como no caso de leito de UTI no Estado da Bahia. A população cresce e o número de vagas não é consideravelmente satisfatório dando margem a judicialização (CARVALHO VF e CARVALHO ER, 2019). No sitio eletrônico do Ministério da Saúde, no Cadastro Nacional de Estabelecimentos de Saúde, constam que no Estado do Tocantins existem 618 leitos e apenas 316 habilitados.

Nessa descrição somam-se os leitos de UTI geral e coronária, adulto e neonatal, bem como os leitos de terapia intensiva para o tratamento da Covid-19. Para os mesmos padrões apresentados, no Estado da Bahia existem 4.384 leitos e 2.063 habilitados (BRASIL, 2020). Assim, a literatura demonstra a existência de 
leitos cadastrados e não habilitados, o que vem de encontro ao resultado encontrado em nossa pesquisa, tendo em vista que se houvesse disponibilidade de leitos de UTI, não haveria a necessidade de judicialização para a busca de leitos de UTI. Assim, nos Estados de Tocantins e Bahia, as solicitações de pareceres foram de 67 e 26 respectivamente. As UTI's estão disponíveis no SUS. Entretanto a oferta ainda é menor em relação à demanda. A situação foi ainda mais agravada pelo atual cenário da pandemia da Covid-19.

Dentre as solicitações de vaga de UTI, embora a grande dificuldade de acesso a UTI para o tratamento da Covid-19 observamos apenas 13 solicitações de pareceres com a descrição deste diagnóstico. Embora não constem dados que possam identificar o resultado positivo para Covid-19 nas outras patologias, não descartamos tal evento. Conforme descreve Fleury S (2012) a judicialização deve ser usada como aliada do SUS, sendo usada como apontador e indicador das deficiências e incentivar para a busca de uma melhoria das políticas de saúde principiando da ciência e de parâmetros sanitários, diminuindo o distanciamento entre o SUS no arcabouço normativo e o SUS executante, como observado pela Coordenadoria de Saúde da DPGE/RJ ao instaurar os procedimentos de insuficiência de leitos de UTI após comprovação do excessivo número de ações ajuizadas no plantão judiciário noturno.

Sobre o perfil na judicialização, destaca-se que em um município de grande porte, observou-se que das 706 ações judiciais, $51,2 \%$ foram impetradas por mulheres acima dos 60 anos de idade. A maior demanda era pelo fornecimento de medicamentos (88,1\%) (BATISTELLA PMF, et al., 2019). Noutro estudo observouse que $65 \%$ dos usuários das ações judiciais eram do sexo feminino, e com uma predominância de idade de 40 a 59 anos $(31,6 \%)$. Embora as mulheres possuam a tendência a buscar o judiciário para serem atendidas, nota-se um perfil diferente. Igualmente, observou-se uma tendência de requerimentos para os medicamentos $(47,4 \%)$ e de medicamentos não disponíveis na tabela SUS (70,2\%) (OLIVEIRA RG e SOUZA AIS, 2014).

Diferente de outros estudos, numa avaliação geral, nós identificamos que 50,96\% das solicitações de pareceres referentes às internações em UTI eram de pacientes do sexo masculino, com uma média de idade de 56 anos. Todavia, numa avaliação por faixa etária, entre 45 a 60 anos de idade, o cenário é diferente, identificando que $30,19 \%$ eram do sexo masculino e $31,37 \%$ referentes ao sexo feminino. A judicialização reivindicando o fornecimento e o acesso a medicamentos e procedimentos médicos começou a ser manifestada a partir da década de 90 , tendo início com as ações que envolviam a solicitação a medicamentos antirretrovirais para a Síndrome da Imunodeficiência Adquirida, a AIDS. Observando-se então a partir daí um aumento gradual do o fornecimento de medicamentos (VENTURA M, et al., 2010).

Em que pese a grande maioria das demandas referem-se a medicamentos e insumos, há intervenções que a urgência do caso, como a busca por leitos de terapia intensiva não seja compatível com o funcionamento desse instrumento de consulta (MARIANO CM, et al., 2018). Ainda que as demandas judiciais venham crescendo observa-se que para alguns autores a judicialização da saúde no âmbito de leitos de UTI no SUS não é a melhor solução (CARVALHO VF e CARVALHO ER, 2019). Talvez essa explicação esteja em consonância com o baixo número de solicitação de pareceres para internação em UTI (104 pareceres, com 80 pareceres favoráveis).

A saúde é um bem tutelado pela CF de 1988, sendo este o bem mais importante ao ser humano. A saúde deve ser abrigada e ofertada pelo Estado, reverenciando o direito à saúde. Deve haver uma entrega de proteção individual e coletiva, bem como o acesso a medicamentos e tratamentos essenciais a preservação e manutenção da vida (RIBAS MC, 2020).

O direito à saúde e seu aspecto fundamental e social, está garantido no artigo 196 e seguintes da CF de 1988. Assim, a saúde, enquanto direito social, apresenta-se meritório no seio jurídico para se aventar a atividade da cidadania e sua preservação enquanto direito humano (CARVALHO VF e CARVALHO ER, 2019).

O acesso à UTI assim como também deve ser feito os pedidos pela via judicial devem ser sob os fundamentos e critérios da qual permitam identificar: a) pacientes que serão beneficiados com a admissão imediata na UTI; e b) pacientes que não serão beneficiados pela terapia intensiva, seja pela condição clínica que não justifica a necessidade de UTI ou pela ausência de perspectiva terapêutica (MOTA GP, 2017). 
Conquanto haja diversas patologias que acometem os indivíduos, notou-se que estavam relacionados a problemas neurológicos (19,23\%) seguido de doenças cardiovasculares (16,35\%). O Conselho Federal de Medicina (CFM) a fim de padronizar os critérios de admissão e alta da UTI, publicou a Resolução CFM $n^{\circ}$ 2.156, de 17 de novembro de 2016 que determina que as admissões em UTI devam ser baseadas em: diagnóstico e necessidade do paciente; serviços médicos disponíveis na instituição; priorização de acordo com a condição do paciente; disponibilidade de leitos e potencial benefício para o paciente com as intervenções terapêuticas e prognóstico (CONSELHO FEDERAL DE MEDICINA, 2016).

Assim sendo, os elementos apresentados pelo Conselho Federal de Medicina são essenciais meios para que o resultado da busca por leito de UTI seja alcançado. Em nossos resultados, identificamos que apenas 47,12\% dos pareceres continham a descrição de Urgência e Emergência apresentada pelo Conselho Federal de Medicina. Essas descrições justificam a necessidade de internação em UTI diante do caso apresentado. De tal modo, os pedidos de internação em UTI não devem ser diferentes daqueles pedidos de medicamentos (laudos médicos e exames, a urgência e a necessidade do pedido). É primordial o esgotamento dos meios administrativos antes de ingressar com o pedido judicial, de modo a ser demonstrado que não há outros meios a se tentar (PAIXÃO AG, et al., 2017).

O impasse de decisões puramente técnicas e solidificadas existe porque o Brasil elegeu a formação tão somente jurídica do magistrado. Para tanto, as ações judiciais que envolvem situações complexas, como por exemplo, a indicação de tratamento, ou ainda o pedido de medicamentos, e não distante o fornecimento de leito de UTI deve o juiz valer-se de conhecimento especializado que possa fundamentar e amparar sua decisão (FORSTER JP, et al., 2020).

Nessas questões, os magistrados podem não possuir o conhecimento técnico necessário de alta complexidade, já que é especializado em Direito e não em na área da Saúde. Por conseguinte, é essencial que ele tenha a sua disposição o parecer de experts que possam abastecer as decisões judiciais das informações técnicas necessárias (FORSTER JP, et al., 2020).

Desse modo, o sistema NATJUS e o e-NATJUS, estruturada pelo Conselho Nacional de Justiça traz aos magistrados um importante dispositivo técnico para aumentar a qualidade do provimento jurisdicional, para que não se baseie apenas em elementos trazidos aos autos pelas partes demandantes e as decisões se tornem cada vez mais técnicas e com menor intervenção de convicções morais e não mais que com elementos objetivos (GONÇALVES JR e NÓBREGA RC, 2020).

A proposta dos Núcleos Técnicos, que foi aplicada pelos Tribunais de Justiça e Tribunais Regionais Federais em todo o País, tem como individualizador o acréscimo da garantia dos magistrados em suas decisões, sem a lentidão ou demora em relação às demandas presentes no Poder Judiciário (MARIANO $\mathrm{CM}$, et al., 2018). Embora as solicitações de pareceres forem tímidas, em especial para a busca de leitos de UTI, e no ano de 2020, com 104 pareceres, talvez a busca por pareceres em detrimento pela busca de outras necessidades, como por exemplo, os medicamentos ainda são maiores.

O apoio técnico cientifico que promove o sistema eletrônico e-NATJUS e o NATJUS nas demandas judiciais vem a acompanhar os recentes fenômenos sociojurídicos (FORSTER JP, et al., 2020). Outrossim, de acordo com o art. 298 do CPC "na decisão que conceder, negar, modificar ou revogar a tutela provisória, o juiz motivará seu convencimento de modo claro e preciso" (BRASIL, 2015). De tal modo, o convencimento deve ser motivado. Assim essa motivação poderá ser baseada em elementos objetivos e sem intervenção de persuasões morais.

\section{CONCLUSÃO}

O processo de judicialização vem se tornando cada vez mais crescente. O sistema NATJUS vem ao encontro de contribuir com uma decisão técnica e fundamentada, em elementos objetivos e literários. Em que pese o baixo número de consultas para a busca de leitos de UTI, essa ferramenta pode ser amplamente apresentada e acrescida, tendo em vista o conhecimento técnico e científico na emissão dos relatórios. Não obstante o baixo número de solicitações de pareceres, compreendemos que as demandas por leitos de UTI são extremamente maiores. Além disso, embora se reconheça a necessidade de internações em leitos de UTI, a oferta do leito, não é perceptível, em relação à alta demanda. 


\section{REFERENCIAS}

1. ADVOCACIA GERAL DA UNIÃO (AGU). MINISTÉRIO DA SAÚDE. Intervenção Judicial na saúde pública: panorama no âmbito da Justiça Federal e apontamentos na seara das justiças estaduais. Brasília, 2012.

2. BATISTELLA PMF, et al. A judicialização na saúde em município de grande porte. 2019 .

3. BRASIL. Portaria no 343212 de agosto de 1998. Estabelece critérios de classificação para as unidades de tratamento intensivo - UTI. 1988.

4. BRASIL. Código de Processo Civil (2015). Código de Processo Civil Brasileiro. Brasília, DF: Senado, 2015.

5. BRASIL. Constituição da República Federativa do Brasil. Brasília. Promulgada em 5 de outubro de 1988.

6. BRASIL. Ministério da Saúde (MS). Cadastro Nacional de Estabelecimentos de Saúde. Secretaria de Atenção à Saúde. 2020

7. CARVALHO VF, CARVALHO ER. A Judicialização da Saúde: A insuficiência de leitos de UTI no Estado da Bahia face aos direitos fundamentais. 2019

8. CONSELHO NACIONAL DE JUSTIÇA (CNJ) 2019. PROVIMENTO № 84, DE 14 DE AGOSTO DE 2019. Dispõe sobre o uso e o funcionamento do Sistema Nacional de Pareceres e Notas Técnicas (e-NatJus) 2019.

9. CONSELHO FEDERAL DE MEDICINA. RESOLUÇÃO (CFM) CFM № 2.156/2016. Estabelece os critérios de admissão e alta em unidade de terapia intensiva. Publicada no D.O.U. de 17 de novembro de 2016, Seção I, p.138-139.

10. COSTA NR. A Disponibilidade de Leitos em Unidade de Tratamento Intensivo no SUS e nos Planos de Saúde Diante da Epidemia da COVID-19 no Brasil. Rio de Janeiro. 2020

11. FLEURY AS. Judicialização pode salvar o SUS. Saúde em Debate, Rio de Janeiro, 2012; 36(93): 159-162.

12. FORSTER JPK, et al. O direito à saúde e a dispensação judicial de medicamentos no Brasil: a ferramenta e-natjus. Revista Derecho y Salud, 2020; 451-63.

13. GONÇALVES JR, NÓBREGA RC. JUDICIALIZAÇÃO DA SAÚDE E A ATUAÇÃO DO NÚCLEO DE APOIO TÉCNICO AO JUDICIÁRIO - NATJUS. Revista do Curso de Direito. Centro Universitário Brazcubas. V4N1: Junho de 2020.

14. LAFFIN NHF, BONACIM CAG. Custos da saúde: judicialização de medicamentos ofertados pelo SUS. XXIV Congresso Brasileiro de Custos - Florianópolis, SC, Brasil, 15 a 17 de novembro de 2017.

15. MADURO LCS, PEREIRA LRL. Processos judiciais para obter medicamentos em Ribeirão Preto. Rev. Bioética, 2020; $28: 1$.

16. MARIANO CM, et al. Diálogos sanitários interinstitucionais e a experiência de implantação do NATJUS. Revista de Investigações Constitucionais, Curitiba, 2018; 5(1): 169-188.

17. MELO EM, et al. Cuidados de enfermagem ao utente sob ventilação mecânica internado em unidade de terapia intensiva. Revista de Enfermagem Referência, Fortaleza, Série IV, 2014; 1: 55-63.

18. MOTA GP. Judicialização do acesso a leitos de UTI no Distrito Federal: dimensões clínica, ética e legal. 2017.

19. OLIVEIRA RG, SOUZA AIS. O perfil das demandas judiciais por direito à saúde pública do Município de Leopoldina-MG. Revista de Saúde Pública do SUS/MG. 2014.

20. PAIM JS. Sistema Único de Saúde (SUS) aos 30 anos. Ciênc. saúde coletiva, Rio de Janeiro, 2018; 23(6): 17231728.

21. PAIXÃO AG, et al. Judicialização do acesso a medicamentos e a leitos de UTI: análise no estado de Goiás. 2017.

22. RIBAS MC. Judicialização de medicamentos no SUS entre 2016 e 2018 em um município da Região Sul do Brasil. 2020. Dissertação (Mestrado em Ciências Sociais Aplicadas) - Universidade Estadual de Ponta Grossa, Ponta Grossa, 2020.

23. RIBEIRO IS, ANDRADE, AC. Judicialização na saúde: Processo Coletivo e Litigância Estratégica. In: III Seminário Internacional em Sociedade e Cultura na Pan-Amazônia - Manaus, 2018.

24. SANTOS FRQ, et al. Resultados adversos de admissões tardias à unidade de terapia intensiva a partir do prontosocorro. Rev. bras. ter. intensiva, São Paulo, 2020; 32(1): 92-98.

25. VENTURA M, et al. Judicialização da saúde, acesso à justiça e a efetividade do direito à saúde. Physis: Revista de Saúde Coletiva, 2010; 20(1): 77-100. 